\title{
SCIENTIFIC REPORTS

\section{Distinct downstream signaling and the roles of VEGF and PIGF in high glucose-mediated injuries of human retinal endothelial cells in culture}

\author{
Wanzhen Jiao ${ }^{1}$, Jia-Fu Ji' ${ }^{2}$, Wenwen $\mathrm{Xu}^{1}$, Wenjuan Bu ${ }^{3}$, Yuanjie Zheng ${ }^{4,5,6,7}$, Aihua $\mathrm{Ma}^{8 *}$, \\ Bojun $\mathrm{Zhao}^{1 *}$ \& Qingfeng Fan ${ }^{9}$
}

Vascular endothelial growth factor (VEGF) and placental growth factor (PIGF) plays a crucial role in breakdown of the blood-retinal barrier due to hyperpermeability in diabetic retinopathy (DR). However, the distinct signaling driven by VEGF and PIGF in the pathogenesis of DR remains unclear. In this study, we investigated VEGF- and PIGF- related signaling pathways and their roles in cultured human microvascular retinal endothelial cells (hRECs) under high glucose conditions (HG; $25 \mathrm{mM}$ ). Apoptotic cell death was evaluated, and FITC conjugated bovine serum albumin across monolayer hRECs served as an index of permeability. Western blots were used to assess the protein levels of VEGF and PIGF, as well as the phosphorylation of p38MAPK, STAT1 and Erk1/2. Knockdown of VEGF and PIGF was performed by using siRNA. Following HG treatment, increases of VEGF and PIGF as well as PKC activity were detected in hRECs. Increased phosphorylations of p38MAPK ${ }^{\text {Thr180/Thr182 }}$, STAT1 ${ }^{\text {Ser727 }}$, and Erk1/2 $2^{\text {Tyr202/Tyr185 }}$ as well as VEGFR1 $1^{\text {Tyr1213 }}$ and VEGFR2 ${ }^{\text {Tyr1175 }}$ were also detected in HG-treated hRECs. Inhibition of PKC activity by Go 6976 prevented HG-induced increases of phosphor-Erk1/2 and nitric oxide synthase (NOS1) expressions as well as hyperpermeability, whereas inhibition of p38MAPK pathway by SB203580 selectively suppressed activation of STAT1 and decreased apoptotic cell death under HG conditions. Moreover, VEGF knockdown predominantly inhibited activation of VEGFR2, and phosphorylation of p38MAPK and STAT1, as well as apoptotic cell death in HG-treated hRECs. Nevertheless, PIGF knockdown mainly suppressed phosphorylation of VEGFR1, PKC, and Erk1/2, as well as NOS1 expressions and hyperpermeability. Taken together, we provide evidence demonstrating that HG-induced elevation of PIGF is responsible for hyperpermeability mainly through increasing activation of PKC-Erk1/2-NOS axis via VEGFR1, while HG-induced elevation of VEGF is associated with induction of apoptotic cell death mainly through increasing activation of p38MAPK/STAT1 signaling via VEGFR2.

Diabetic retinopathy (DR), one of the most prominent microvascular complications of diabetes mellitus, is the leading cause of new-onset blindness in the developed countries ${ }^{1,2}$. It was reported that across China, the prevalence of DR and sight-threatening DR was $27.9 \%$ and $12.6 \%$ in diabetes, respectively ${ }^{3}$. DR is classified as either non-proliferative DR, which is characterized by microaneurysms and intraretinal hemorrhage, or proliferative DR, which has been identified by vitreous hemorrhage and neovascularization of the eye fundus and iris ${ }^{1}$. Generally, it has been accepted that angiogenesis and inflammation crosstalk play an essential role in the

\footnotetext{
${ }^{1}$ Department of Ophthalmology, Shandong Provincial Hospital Affiliated to Shandong University, Jinan, 250021, China. ${ }^{2}$ Department of Anesthesiology, Affiliated Hospital of Shandong University of Traditional Chinese Medicine, Jinan, 250014, China. ${ }^{3}$ Department of Ophthalmology, Jining First People's Hospital, Jining, 272011, China. ${ }^{4}$ School of Information Science and Engineering of Shandong Normal University, Jinan, 250014, China. Institute of Biomedical Sciences of Shandong Normal University, Jinan, 250014, China. ${ }^{6}$ Key Lab of Intelligent Computing \& Information Security of Shandong Normal University, Jinan, 250014, China. ${ }^{7}$ Shandong Provincial Key Laboratory for Distributed Computer Software Novel Technology, Jinan, 250014, China. ${ }^{8}$ Department of Pediatrics, Shandong Provincial Hospital Affiliated to Shandong University, Jinan, 250021, China. ${ }^{9}$ Renal-Electrolyte and Hypertension Division, Perelman School of Medicine of University of Pennsylvania, Philadelphia, PA, 19104, USA. *email: aihuama@hotmail.co.uk; 15168860708@163.com
} 
pathogenesis of $\mathrm{DR}^{4-6}$. Diabetes and hyperglycemia have obvious effects on vascular endothelial cell permeability, adhesion and proliferation. Increased permeability results in vascular leakage and occlusions, as well as angiogenesis $^{4-6}$.

Angiogenesis is a complex process that is mediated by various growth factors including vascular endothelial growth factor (VEGF) family ${ }^{7}$. VEGF family consists of seven secreted dimeric proteins, VEGF-A, B, C, D, E, F and the placental growth factor (PlGF). VEGF binds to at least two transmembrane tyrosine kinase receptors, named Flt1 (VEGF receptor-1 or VEGFR1) and Flk-1/KDR (VEGF receptor-2 or VEGFR2), on endothelial cells ${ }^{8}$. The levels of aqueous and vitreous VEGF-A were significantly elevated in patients with proliferative DR compared to the non-proliferative DR ${ }^{9}$. Unlike VEGF-A, VEGF-B possesses low angiogenic potential and does not induce vessel formation or sprouting. Signaling via VEGFR1 and neutropilin 1 (NRP-1), VEGF-B was considered as a potent survival factor of vascular cells which keeps the neo-vessels from apoptosis ${ }^{10,11}$. PlGF, a member belonging to the VEGF family, was originally isolated from the human placenta and directly signals through VEGFR1. It was reported that the levels of PlGF were elevated in the vitreous and aqueous humor of patients with $\mathrm{DR}^{12,13}$. A comparative research of vitreous PlGF levels in proliferative DR patients treated with or without anti-VEGF agent therapy revealed that PlGF levels were highly correlated with VEGF-A levels in active proliferative DR. This suggested that PlGF may also involve angiogenesis in the pathogenesis of DR possibly by amplifying the role of VEGF-A ${ }^{14}$. Some studies showed that stimulation of monocytes with PlGF or VEGF-A induced activation of several intracellular signaling molecules including phosphatidylinositol-3 kinase (PI3K), protein kinase B (Akt), extracellular signal-regulated kinase-1/2 (Erk1/2), and p38 mitogen-activated protein kinases (MAPK) ${ }^{15-17}$. Overproduction of nitric oxide synthase (NOS) induced by activated PKC is related to vasodilation and hyperpermeability ${ }^{18}$. STAT1 (signal transducer and activator of transcription 1) has been implicated as a mediator of a variety of biological responses such as apoptosis in response to stimulations of specific growth factors and cytokines $^{19}$. However, the exact roles of VEGF and PlGF, and their distinct downstream signaling have not been understood completely in the pathogenesis of angiogenesis of DR.

The present study aims to investigate the distinct signaling pathways and their roles of VEGF and PlGF in high glucose (HG)-induced injuries of human microvascular retinal endothelial cells (hRECs). We demonstrated that in HG-treated hRECs, i) the abundances of both VEGF and PlGF were increased significantly, ii) VEGF-mediated activation of p38MAPK/STAT1 signaling via selectively binging to VEGFR2 mainly led to induction of apoptosis, and iii) PlGF-induced activation of PKC/Erk1/2/NOS1 pathway via selectively binding to VEGFR1 mainly resulted in hyperpermeability.

\section{Results}

Injuries of hREC in high glucose conditions. A key manifestation of DR is macular edema which is mainly caused by increased microvascular permeability ${ }^{20}$. In this study, the permeability of monolayer hRECs growing on Transwell filters was evaluated by using FITC-conjugated bovine serum albumin (BSA). Compared to the mannitol (MN) osmotic control, HG caused a time-dependent increase of permeability, showing a significant $(p<0.05)$ hyperpermeability at $24 \mathrm{~h}$, persisting to $72 \mathrm{~h}$ (Fig. 1a). We also evaluated the uptake of BSA by using western blot assay. The amounts of BSA were increased significantly $(p<0.05)$ at 24 and $48 \mathrm{~h}$ in the cellular lysates isolated from HG but not MN treated hRECs (Fig. 1b). These findings demonstrated that hyperpermeability of hRECs was induced under HG conditions. In addition, as reported previously ${ }^{21}$, increased apoptotic cell death was remarkably $(p<0.05)$ detected at $48 \mathrm{~h}$ in $\mathrm{HG}$ treated cells in comparison with the controls (Fig. 1c). Moreover, increased active caspase 3 level was detected significantly $(p<0.05)$ at 24 and $48 \mathrm{~h}$, whereas the anti-apoptotic protein Bcl-2 showed a significant $(p<0.05)$ reduction in HG treated hRECs (Fig. 1d). Therefore, these findings suggest that injuries of hRECs under HG conditions present with increased apoptosis and hyperpermeability.

Activation of the PKC-Erk1/2-NOS1 axis is related to hyperpermeability in HG-treated hRECs. In HG-treated hRECs, the protein level of protein kinase C (PKC) was increased significantly $(p<0.05)$ at 24 and $48 \mathrm{~h}$ (Fig. 2a). Following HG treatment, time-dependent increases of phospho-Erk1/2 at Tyr202/Tyr185 and NOS1 expression were detected ( $p<0.05$ ), respectively (Fig. 2b,c). To investigate the role of PKC, Go 6976, a specific inhibitor of PKC activity was applied at 3 different concentrations. In normal cultured hRECs, the application of $500 \mathrm{nM}$ of Go 6976 almost completely suppressed activation of PKC at $48 \mathrm{~h}$. In this study, an increase of PKC activity was significantly $(p<0.05)$ detected at $48 \mathrm{~h}$ in HG-treated hRECs, which was significantly $(p<0.05)$ suppressed by Go 6976 at the concentration of $500 \mathrm{nM}$ (Fig. 2d).

Interestingly, western blot assay revealed that HG-induced increases of phospho-Erk1/2 ${ }^{\text {Tyr202/Tyr185 }}$ and NOS1 expression at $48 \mathrm{~h}$ were significantly $(p<0.05)$ inhibited by the application of $500 \mathrm{nM}$ Go 6976 . But, the phosphorylated Erk1/2 level and NOS1 expression were still higher $(p<0.05)$ in HG-treated hRECs with Go 6976 than the controls (Fig. 2e). This suggests that HG mediated activation of Erk1/2-NOS1 signaling partially through upregulation of PKC activity. We also studied the effects of inhibition of PKC activity on permeability and apoptosis of hRECs under HG conditions. The presence of $500 \mathrm{nM}$ Go 6976 significantly $(p<0.05)$ decreased the permeability of FITC-albumin, but did not affect apoptotic level in hRECs treated with HG for $48 \mathrm{~h}$ (Fig. 2f,g). These findings demonstrate that overactivation of the PKC-Erk1/2-NOS1 signaling plays a critical role in regulating the permeability of hRECs under HG conditions.

Activation of the P38MAPK/STAT1 pathway is related to apoptosis induction in HG-treated hRECs. It has been well known that activation of the p38MAPK signaling plays an important role in inducing apoptosis in various cell types ${ }^{22}$. In this study, rapid increase of phospho-p38MAPK at Thr180/Tyr182 was significantly $(p<0.05)$ detected at 5,10 , and 30 min following HG treatment. In comparison with the dynamics of p38MAPK activation, a delay increase of phospho-STAT1 at Ser727 was significantly $(p<0.05)$ revealed at 10 and $30 \mathrm{~min}$ in HG-treated hRECs (Fig. 3a). SB203580, a specific p38MAPK inhibitor, was applied to inhibit activation 


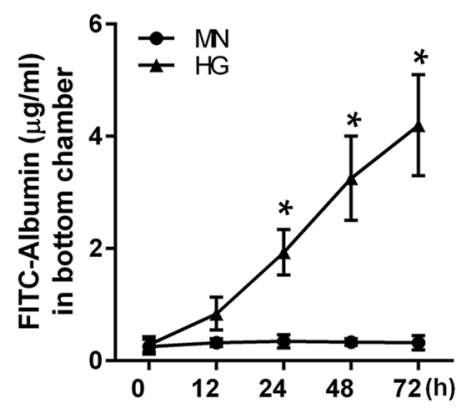

b

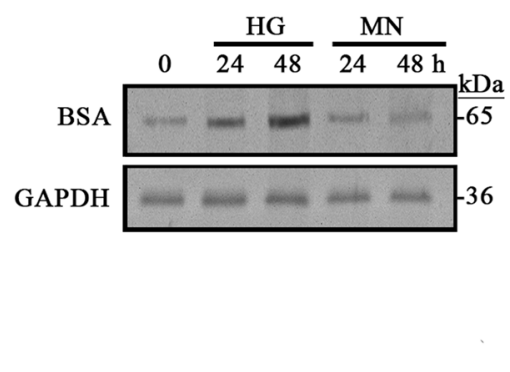

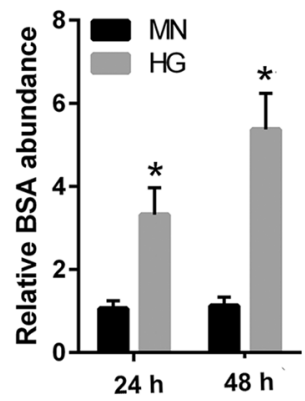

$\mathrm{c}$

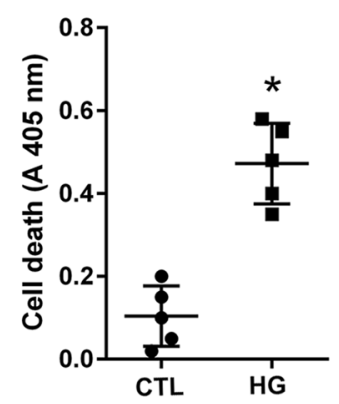

d
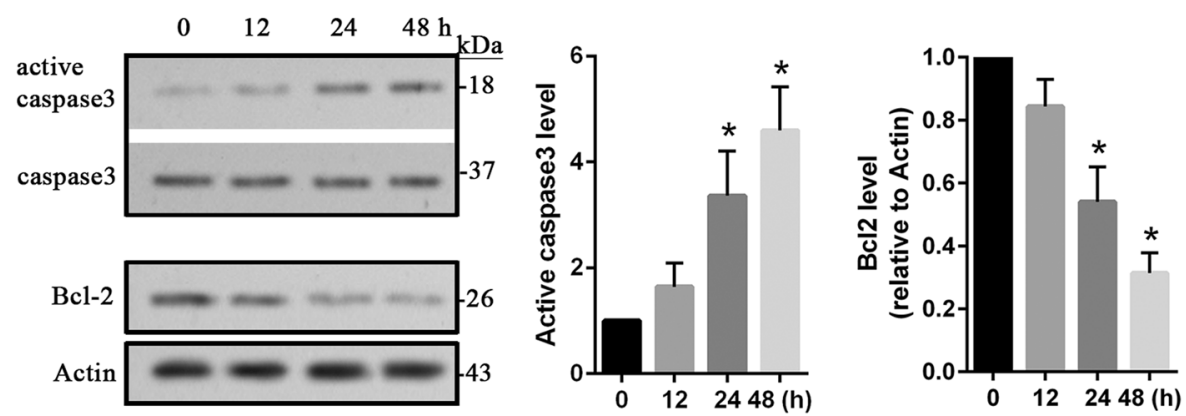

Figure 1. HG induces injuries in in vitro cultured hRECs. (a). hRECs were grown on Transwell filters. Cells were then treated with high glucose $(\mathrm{HG}, 25 \mathrm{mM})$ or mannitol $(\mathrm{MN})$ as the osmotic control for different time periods. The permeability of monolayer cells was evaluated using FITC-conjugated albumin. Data are presented mean $\pm S D$. $n=3$. ${ }^{*} p<0.05 v s 0 h$. (b). hRECs were grown in 6-well plate, and treated for 24 and $48 \mathrm{~h}$ with HG or MN, respectively. $10 \mu \mathrm{g} / \mathrm{ml}$ of bovine serum albumin (BSA) was then added and incubated for $30 \mathrm{~min}$. After 3 washes, cells were lysed and total protein was extracted for immunoblotting with anti-BSA antibody. Data are presented mean $\pm S D . n=3$. $* p<0.01 v s M N$. (c). hRECs were treated for $24 \mathrm{~h}$ with HG. Apoptotic cell death was determined by using the Cell Death Detection ELISA. Data are presented mean $\pm S D . n=5$. *: $p<0.05$ vs control (CTL). (d). hRECs were treated with HG for the indicated time periods. Total cellular protein was extracted, and immunoblotting assay was performed for detection of activated caspase 3 and Bcl-2. MN-treated hRECs were used as the treatment control (Supplementary Fig. 3). Data are presented mean $\pm S D . n=3 . * p<0.01$ vs 0 h. Full length blots of $b$ and $d$ are provided in Supplementary Fig. 4.

of p38MAPK signaling. HG-induced increase of phosphorylated p38MAPK was significantly $(p<0.05)$ prevented by the application of $10 \mu \mathrm{M}$ SB203580 (Fig. 3b). It was reported that p38MAPK is able to phosphorylate STAT1 at Ser727 in vitro ${ }^{23}$. As expected, upregulation of phosphorylated STAT1 was significantly $(p<0.05)$ inhibited by $10 \mu \mathrm{M}$ SB203580 in HG-treated hRECs (Fig. 3b). We also explored the effects of p38MPAK inhibition on permeability and apoptosis in HG-treated hRECs. Interestingly, SB203580 remarkably $(p<0.05)$ decreased HG-induced apoptotic cell death, but did not show effect on hyperpermeability of FITC-albumin (Fig. 3c,d). These findings show that overactivation of the p38MAPK/STAT1 pathway is required at least in part for induction of apoptotic cell death of hRECs under HG conditions.

Abundances of VEGF and PIGF are increased in HG-treated hRECs. Here, the abundances of VEGF and PlGF were measured at different time points in HG-treated hRECs. The mRNA levels of VEGF and PlGF were quantitatively assessed by using real time RT-PCR, showing a time-dependent increase $(p<0.05)$ starting at $12 \mathrm{~h}$ in HG-treated hRECs (Fig. 4a). We also measured the amounts of secreted VEGF and PlGF by using ELISA assay in the cultured media. Significant increase was detected $(p<0.05)$ since $12 \mathrm{~h}$ following HG treatment (Fig. 4b). Moreover, the abundances of VEGF and PlGF proteins were significantly $(p<0.05)$ upregulated at 24 and $48 \mathrm{~h}$ following HG treatment (Fig. 4c). These data suggest that both expressions and secretions of VEGF and PlGF were remarkably increased under HG conditions.

VEGF predominantly activates the p38MAPK/STAT1 signaling via VEGFR2 and thus induces apoptotic cell death in HG-treated hRECs. VEGF family and its receptors are vitally involved in the process of angiogenesis ${ }^{8}$. To specify the downstream signaling of VEGF, we deleted VEGF expressions by using 3 different concentrations of siRNA that is specifically targeted to human VEGF-A. Western blot assay shows a significant $(p<0.05)$ dose-dependent reduction of VEGF in HG-treated hRECs expressing siVEGF-A (Fig. 5a). Interestingly, the levels of both phospho-VEGFR1 at Tyr1213 and phospho-VEGFR2 at Tyr1175 were significantly $(p<0.05)$ increased following HG-treatment. However, increased phospho-VEGFR2 ${ }^{\text {Tyr1175 }}$,

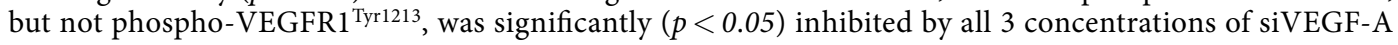


a

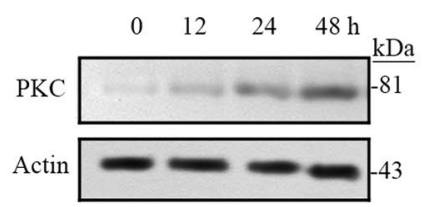

c
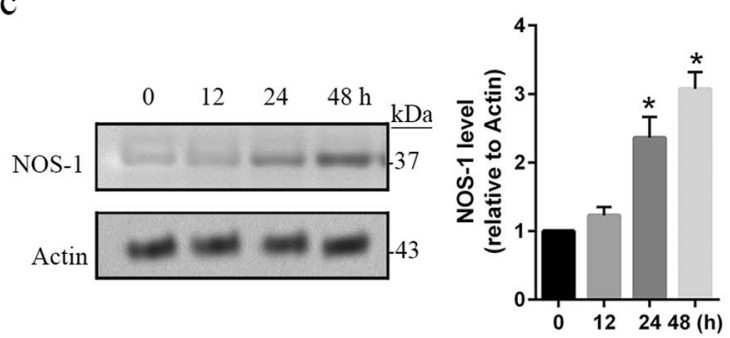

$\mathrm{e}$
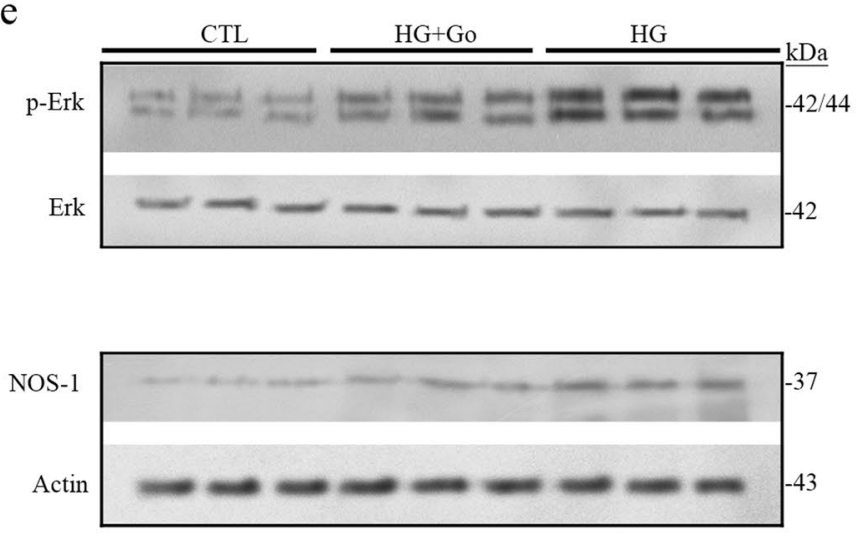

$\mathrm{f}$

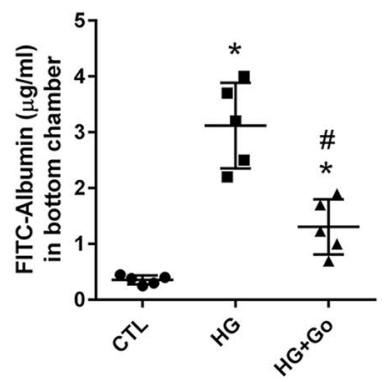

$\mathrm{b}$
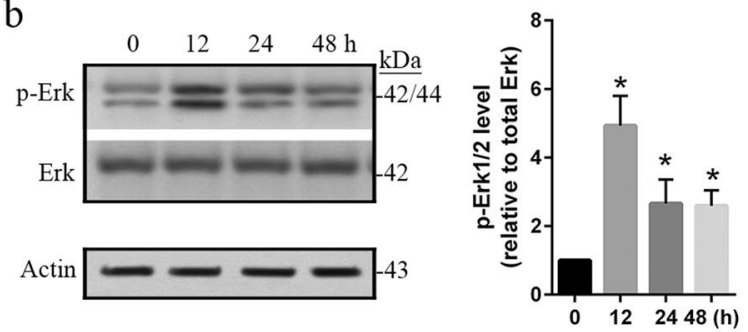

d
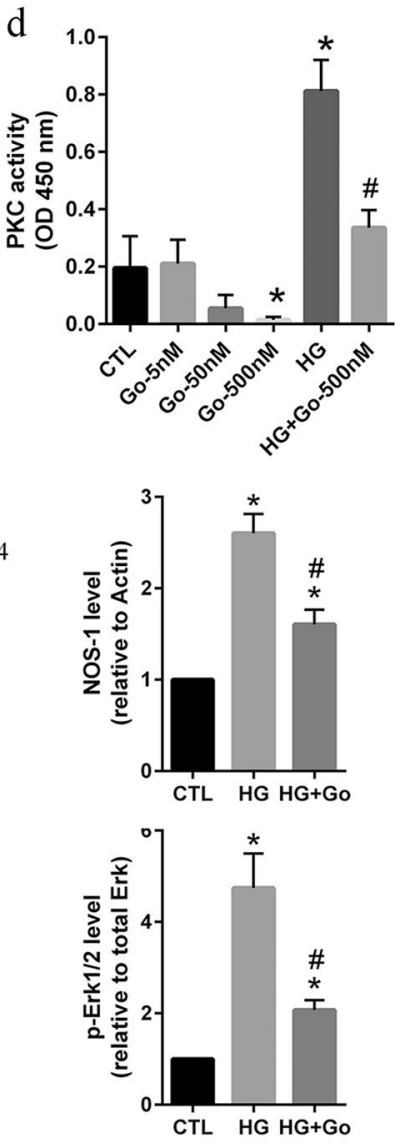

g

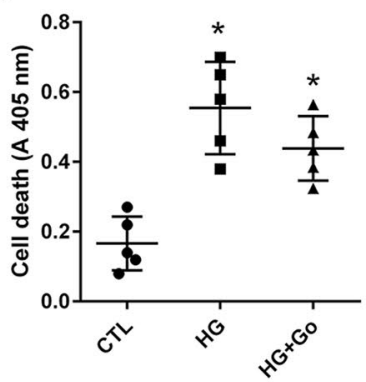

Figure 2. Activation of the PKC-Erk1/2-NOS1 axis is related to hyperpermeability in HG-treated hRECs. (a-c) hRECs were treated with high glucose $(\mathrm{HG}, 25 \mathrm{mM})$ as the indicated time periods. MN-treated hRECs were used as the treatment control (Supplementary Fig. 5a). Cells were then lysed and total protein was extracted for immunoblotting with anti-PKC (a), anti-phospho-Erk1/2 $2^{\text {Tyr202/Tyr185 }}(\mathbf{b})$ and anti-NOS1 (c) antibodies. Data are presented mean $\pm S D . n=3$. ${ }^{*} p<0.05 v s 0 h$. (d). hRECs were cultured for $24 \mathrm{~h}$ under HG conditions in the presence or absence of PKC inhibitor Go 6976. Total cellular protein was extracted, and $50 \mu \mathrm{g}$ was used for evaluation of PKC kinase activity. Data are presented mean $\pm S D . n=3$. ${ }^{*} p<0.05 v s C T L ;{ }^{*} p<0.05 v s$ HG. (e). hRECs were cultured for $24 \mathrm{~h}$ in HG with or without Go 6976 (500 nM). Cells were then lysed and total protein was extracted for immunoblotting with anti-phospho-Erk1/2 ${ }^{\text {Tyr202/Tyr185 }}$ and anti-NOS1 antibodies. The effects of Go 6976 on Erk activation were explored in hRECs under normoglycemia condition (Supplementary Fig. 5b). Data are presented mean $\pm S D . n=3$. ${ }^{*} p<0.05 v s C T L$. (f). Cells were grown on Transwell filters, and treated for $24 \mathrm{~h}$ in HG with or without Go $6976(500 \mathrm{nM})$. The permeability of monolayer cells was evaluated using FITC 
conjugated albumin. (g). hRECs were cultured for $24 \mathrm{~h}$ in HG with or without Go 6976 (500 nM). Apoptotic cell death was determined using the Cell Death Detection ELISA. f and g. Data are presented mean $\pm S D$. $n=5$. $* p<0.01$ vs CTL; ${ }^{*} p<0.05$ vs HG. Full length blots of $a, b, c$ and $e$ are provided in Supplementary Fig. 6 . In $b$ and e, stripped membranes from phospho-Erk were reblotted for total Erk.

(Fig. 5a). Increase of the phosphorylated VEGFR $1^{\text {Tyr1213 }}$ level was slightly inhibited in HG-treated hRECs that only expressed high concentrations of siVEGF-A. These data suggest that in cultured hRECs exposed to HG conditions, increased VEGF mainly activated VEGFR2. In addition, knockdown of VEGF did not have significant $(p>0.05)$ influence on PKC activity in HG-treated hRECs (Fig. 5b). Moreover, HG-induced increases of phospho-Erk1/2 $2^{\text {Tyr202/Tyr185 }}$ were not affected by VEGF knockdown (Fig. 5c). The level of NOS1 was significantly $(p<0.05)$ decreased in HG-treated hRECs expressing siVEGF-A compared to HG-treated cells expressing siCTL, although it was still higher than nontreated cells (Fig. 5c). However, HG-increased phospho-p38MAPK Tyr180/Tyr182 and phospho-STAT1 ${ }^{\text {Ser727 }}$ was significantly $(p<0.05)$ suppressed by VEGF knockdown (Fig. $\left.5 \mathrm{~d}\right)$. In consistent, VEGF knockdown significantly $(p<0.05)$ decreased apoptotic cell death (Fig. 5e), but did not show obvious effects on permeability in HG-treated hRECs (Fig. 5f). These findings demonstrate that VEGF selectively activated VEGFR2 by enhancing its phosphorylation at Tyr1175, thus inducing apoptotic cell death through p38MAPK/STAT1 signaling pathway.

PIGF mainly activates the PKC-Erk1/2-NOS1 axis via VEGFR1 responsible for hyperpermeability in HG-treated hRECs. PlGF belongs to the VEGF family, and its level was elevated in the vitreous and aqueous humor of patients with $\mathrm{DR}^{12-14}$. Here, knockdown of PlGF was obtained by introducing 3 different concentrations of siPlGF specifically targeted to human PlGF in HG-treated hRECs. The results showed that HG-induced increase of PlGF was dramatically $(p<0.05)$ suppressed by siPlGF (Fig. 6a). We then investigated the effects of PlGF knockdown on phosphorylated VEGFR1 and phosphorylated VEGFR2 level. Our data show that increased phospho-VEGFR1 $1^{\text {Tyr1213 }}$, but not phospho-VEGFR2 ${ }^{\text {Tyr1175, }}$, was remarkably $(p<0.05)$ inhibited by knockdown of PIGF in HG-treated hRECs compared to HG alone and HG-treated cells with siCTL (Fig. 6a). This suggests that PlGF may predominantly activate VEGFR1 following HG stimulations in hRECs. Knockdown of PlGF showed significant $(p<0.05)$ inhibition on PKC activity in HG-treated hRECs (Fig. 6b). Moreover,

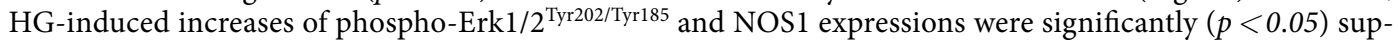
pressed by PIGF knockdown (Fig. 6c). Compared to nontreated cells, the level of phosphorylated Erk1/2 was still higher $(p<0.05)$ in HG-treated hRECs with PlGF knockdown (Fig. $6 \mathrm{c}$ ), suggesting that other signal pathway may involve Erk1/2 activation in hRECs under HG conditions. However, increased phospho-p38MAPK Tyr180/Tyr182 and phospho-STAT $1^{\text {Ser727 }}$ was not affected by PIGF knockdown in HG-treated hRECs (Fig. 6d). In consistent, PIGF knockdown did not show obvious effects on apoptosis (Fig. 6e); but, significantly $(p<0.05)$ decreased permeability in HG-treated hRECs (Fig. 6f).

\section{Discussion}

Proliferative DR is the advanced stage of DR, characterized by retinal neovascularization and hyperpermeability, hemorrhaging pre-retinal neovessels and potential vitreal contraction, and retinal detachment ${ }^{24,25}$. Deterioration of the blood-retinal barrier results in extravasation of fluids from permeable capillaries, leading to diabetic macular edema ${ }^{10}$. It has been reported that several factors including elevated levels of VEGF and PlGF involve breakdown of the blood-retinal barrier in $\mathrm{DR}^{10}$. In the present study, we demonstrated that in cultured hRECs under HG conditions, elevated VEGF and PIGF significantly increased endothelial cell permeability and apoptotic cell death by distinct downstream signaling PKC/Erk/NOS and p38MAPK/STAT1 pathways, respectively. A potential concern is whether PlGF-induced hyperpermeability results from a loss of cells under HG condition. Actually, we did not observe significant detachment of hRECs following HG treatment. Moreover, confluent monolayer cells were used for Transwell assay, and the western blot assay also detected increased BSA uptake in HG-treated hRECs. Therefore, PlGF-induced hyperpermeability may not result from a loss of cells in HG-treated hRECs. However, it should be noted here that the FITC-BSA and Transwell assays may not be sufficient to assess the endothelial permeability. More accurate methods such as trans-endothelial electrical resistance (TEER) and electrical cell-substrate impedance sensing (ECIS) should be used in the future for permeability analysis.

As described previously ${ }^{26}$, we detected increases of the mRNA and protein levels of VEGF and PlGF as well as the concentrations of secreted VEGF and PlGF in HG-treated hRECs. Binding of VEGF to VEGFR2 led to activation of PI3K and phospholipase C- $\gamma$, which then activated PKC and the release of free calcium from internal stores ${ }^{27}$. In this study, increased activation of PKC was detected in cultured hRECs under HG conditions. Inhibition of PKC activation predominantly decreased permeability; but, did not have remarkable influences on apoptotic cell death. Studies with specific inhibitors revealed a role for the ERK1/2 in VEGF-induced hyperpermeability ${ }^{28}$. Growth factors are thought to activate ERK1/2 through the Ras-Raf-MEK pathway ${ }^{29}$. However, it was found that VEGF can induce Ras-independent ERK1/2 activation in which PKC involved ${ }^{30}$. Here, increased phosphorylation of Erk1/2 and NOS1 expressions were detected in HG-treated hRECs, which was prevented by the inhibition of PKC. Increased activity of PKC can result in activation of endothelial NOS, leading to NO release $\mathrm{e}^{31}$. It has been reported that increased NO is related to vasodilation and hyperpermeability ${ }^{27}$. In cultured human umbilical vein endothelial cell, ERK1/2 and NOS are elements of different signaling pathways that are associated with VEGF-induced hyperpermeability ${ }^{32}$. Therefore, our findings suggest that HG-induced hyperpermeability may be mainly mediated by the activation of PKC-Erk1/2-NOS axis in cultured hRECs.

Under HG conditions, it has been reported that p38MAPK and Erk1/2 is activated in hRECs ${ }^{26,33,34}$. Increased serine phosphorylation of transcription factor STAT1 followed by activation of p38MAPK plays an important 
$\mathrm{a}$

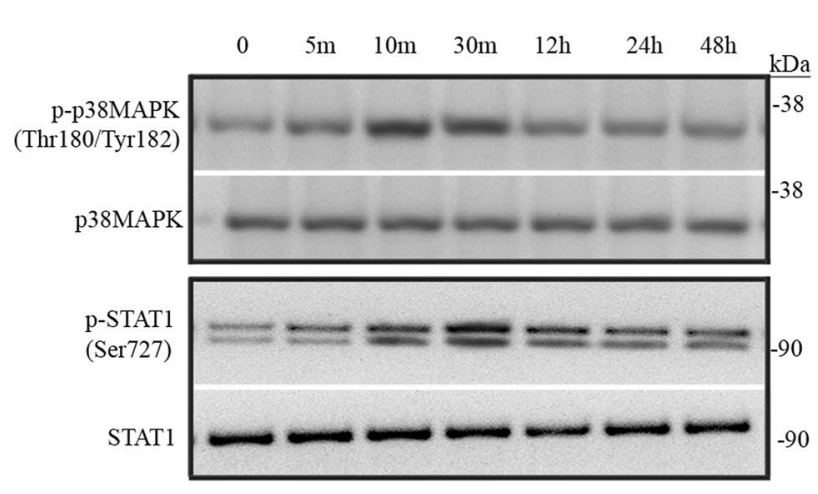

b

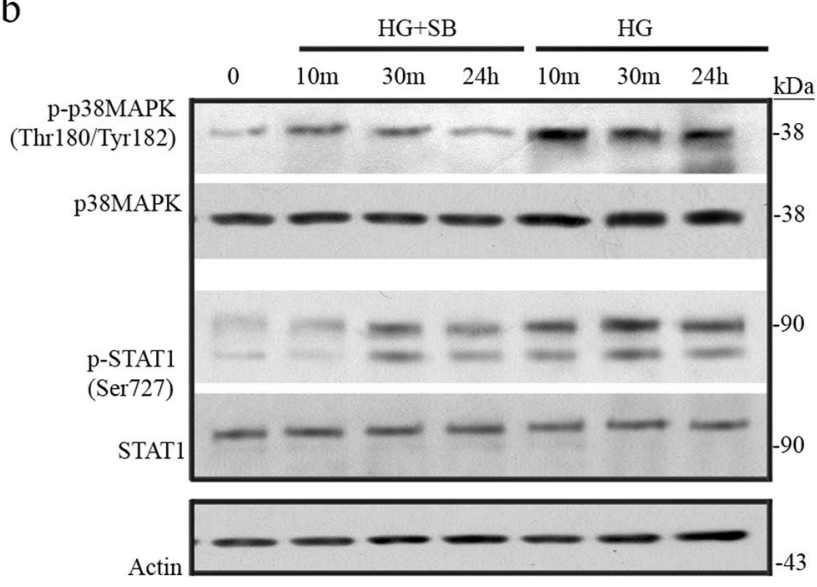

$\mathrm{C}$

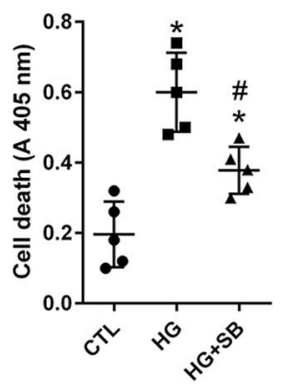

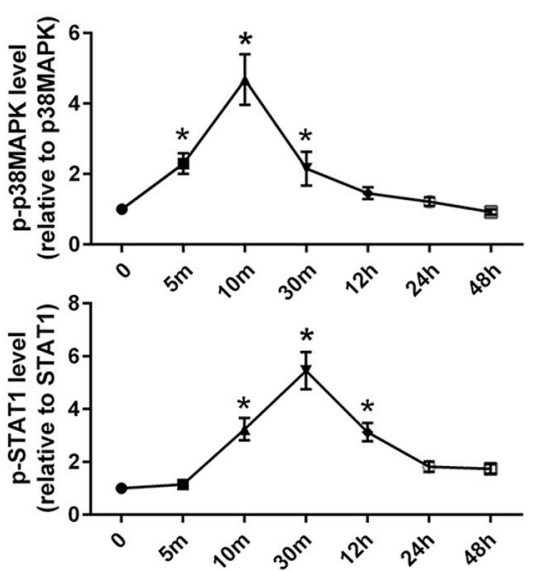
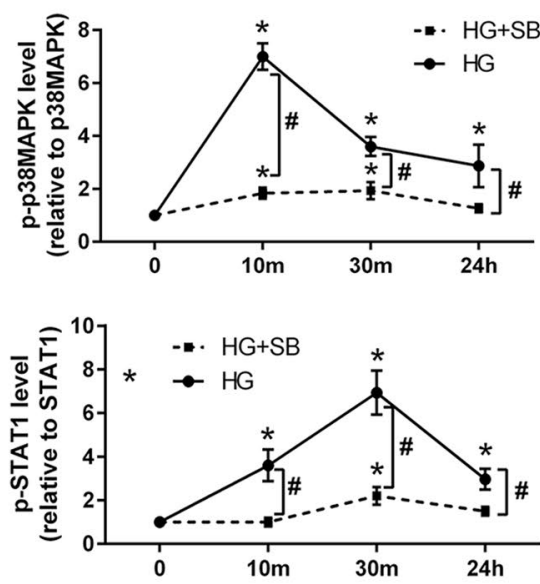

d

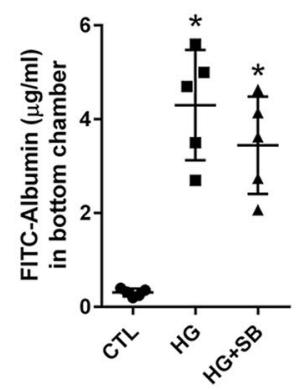

Figure 3. Activation of the p38MAPK/STAT1 signaling mediates apoptosis in HG-treated hRECs. (a). hRECs were treated with high glucose $(\mathrm{HG}, 25 \mathrm{mM})$ for the indicated time periods. Cells were lysed and total protein was extracted for immunoblotting with anti-phospho-p38 MAPK Tyr180/Tyr182 and anti-phospho-STAT1 ${ }^{\text {Ser727. }}$. MN-treated hRECs were used as the treatment control (Supplementary Fig. 7a). Data are presented mean $\pm S D$. $n=3$. ${ }^{*} p<0.05 v s 0 h$. (b). hRECs were treated for the indicated time periods with HG in the presence or absence of p38 MAPK inhibitor SB203580 $(10 \mu \mathrm{M})$. Cells were lysed and total protein was extracted for immunoblotting with anti-phospho-p38MAPK ${ }^{\text {Tyr180/Tyr182 }}$ and anti-phospho-STAT1 ${ }^{\text {Ser727. The effects of }}$ SB203580 on STAT1 activation were explored in hRECs under normoglycemia condition (Supplementary Fig. 7b). Data are presented mean $\pm S D . n=3$. ${ }^{*} p<0.05 v s 0 h ;{ }^{*} p<0.05, H G v s H G+S B$ (c). hRECs were treated for $24 \mathrm{~h}$ with $\mathrm{HG}$ in the presence or absence of SB203580 $(10 \mu \mathrm{M})$. Apoptotic cell death was determined using the Cell Death Detection ELISA. (d). Cells were grown on Transwell filters and treated for $24 \mathrm{~h}$ with HG in the presence or absence of SB203580 $(10 \mu \mathrm{M})$. The permeability of monolayer cells was evaluated using FITC conjugated albumin. $c$ and $d:$ Data are presented mean $\pm S D . n=5 .{ }^{*} p<0.05$ vs CTL; ${ }^{\#} p<0.05$ vs HG. Full length blots of $a$ and $b$ are provided in Supplementary Fig. 8. In a and $b$, stripped membranes from phospho-p38MAPK and phosphor-STAT1 were reblotted for total p38MAPK and STAT1, respectively. 


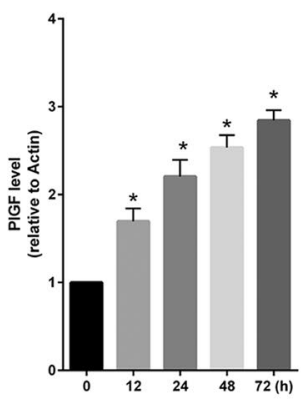

c

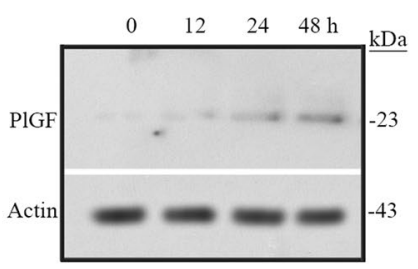

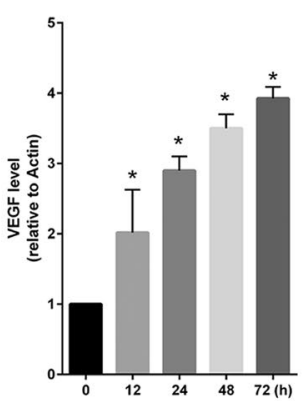

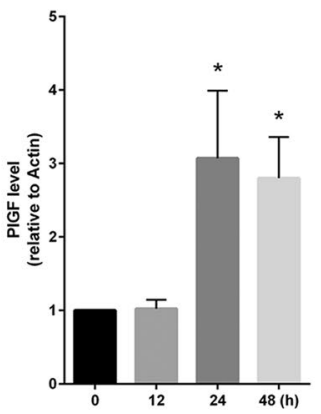

$\mathrm{b}$
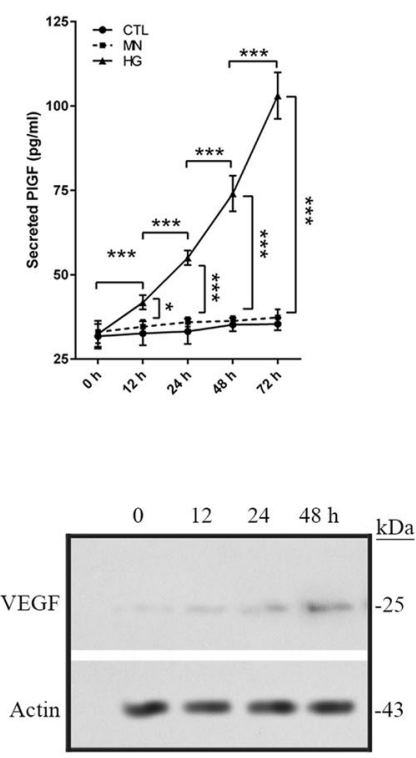
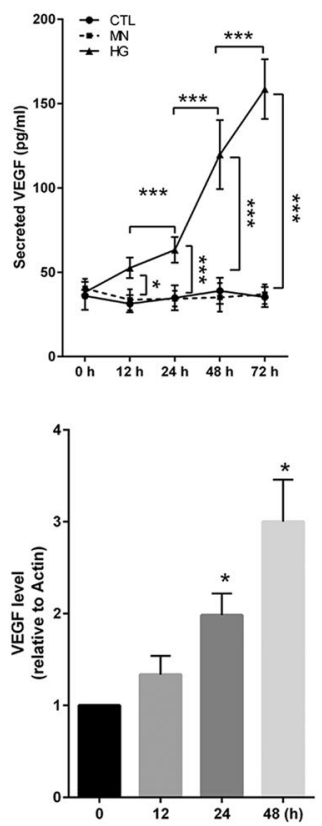

Figure 4. The abundances of PlGF and VEGF are increased in HG-treated hRECs. hRECs were treated with high glucose (HG, $25 \mathrm{mM}$ ) for the indicated time periods. Non-treated cell (CTL) or mannitol (MN) treated cell was used as the controls. (a). Quantitative real PCR was performed to assess the mRNA levels of VEGF and PIGF. (b). Culture medium was collected and ELISA was performed to quantitate the amount of secreted PlGf and VEGF, respectively. (c). Cells were lysed and total protein was extracted for immunoblotting with anti-PlGF and anti-VEGF antibodies. Full length blots are shown for PlGF and VEGF. MN-treated hRECs were used as the treatment control (Supplementary Fig. 9). Data are presented mean $\pm S D . n=3 . b: * p<0.05, * * p<0.01$, $* * * p<0.001$. n.s. non-significance. $a$ and $c: * p<0.01$ vs $0 \mathrm{~h}$.

role in apoptosis induction in HeLa contaminant WISH cell ${ }^{35}$. Here, increased phosphorylation of p38MAPK at Tyr180/Tyr182 and STAT1 at Ser127 were detected in HG-treated hRECs. Inhibition of p38MAPK significantly suppressed phospho-STAT1 level and apoptotic cell death; but did not show remarkable influences on permeability in HG-treated hRECs. These findings suggest that overactivation of p38MAPK/STAT1 signaling may preferably induce apoptotic cell death in HG-treated hRECs. In addition, as previously reported ${ }^{26,33,34}$, increased activation of Akt was also detected in our HG-treated hRECs (data not shown). However, inhibition of PKC and p38MAPK did not alter phospho-Akt level in HG-treated hRECs (data not shown), suggesting that Akt activation may be independent on PKC and p38MAPK signaling pathway.

In hRECs exposed to VEGF and HG, VEGF upregulated PlGF expression via the MAPK signaling pathway and partially through $\mathrm{PKC}^{36}$. It was found that fibroblast growth factor (FGF2)-mediated enhancement of PlGF expression was dependent on VEGF function ${ }^{37}$. Therefore, a potential crosstalk is present between VEGF and PlGF signaling. To separate the distinct role of VEGF and PlGF in HG-induced injuries of hRECs, we firstly assessed the phosphorylation levels of VEGFR1 and VEGFR2, and then performed knockdown assay by using the siRNA that specifically targeted against VEGF-A and PlGF, respectively. Here, we detected significant upregulation of the phosphorylated VEGFR1 at Tyr1213 and VEGFR2 at Tyr1175 following HG treatment. Some studies reported that the expression of VEGFR1 and VEGFR2 is upregulated in the retinal vessels of diabetic rats ${ }^{38}$. However, our results show that knockdown of PlGF significantly decreased the tyrosine phosphorylation level of VEGFR1 but not VEGFR2 in HG-treated hRECs, suggesting that PlGF may mainly transduce downstream signaling via activation of VEGFR1 in cultured hRECs under HG conditions. The strong correlation of PlGF levels with proliferative DR disease status and expression of VEGFR1 was reported in human proliferative DR ${ }^{14}$. Interestingly, knockdown of PlGF dramatically prevented HG-induced increase of PKC activity. In placenta cell lines exposed to HG, the expressions of PlGF and VEGF as well as PKC $\beta$ activity were also increased significantly $^{39}$. Here, we found that increased phospho-Erk1/2 and NOS1 expressions were significantly inhibited by knockdown of PlGF in HG-treated hRECs. Consistently, knockdown of PlGF decreased permeability; but, did not affect the level of apoptotic cell death under HG conditions. Our findings suggest that PlGF mainly increased the activation of PKC-Erk1/2-NOS axis via VEGFR1, thus leading to hyperpermeability in HG-treated hRECs (Fig. 7). However, stimulations of immortalized bovine RECs with various growth factors showed that VEGF-A, but not PlGF, impairs the barrier function of bovine RECs, and that activation of VEGFR2, probably in concert with neuropilin-1, seems to be sufficient to induce barrier dysfunction ${ }^{40}$. Therefore, the addition of PlGF or VEGF to hRECs treated with or without HG may provide more important information. This could help us further understand the role and downstream signaling of PlGF and VEGF in retinal endothelial cell injuries. 


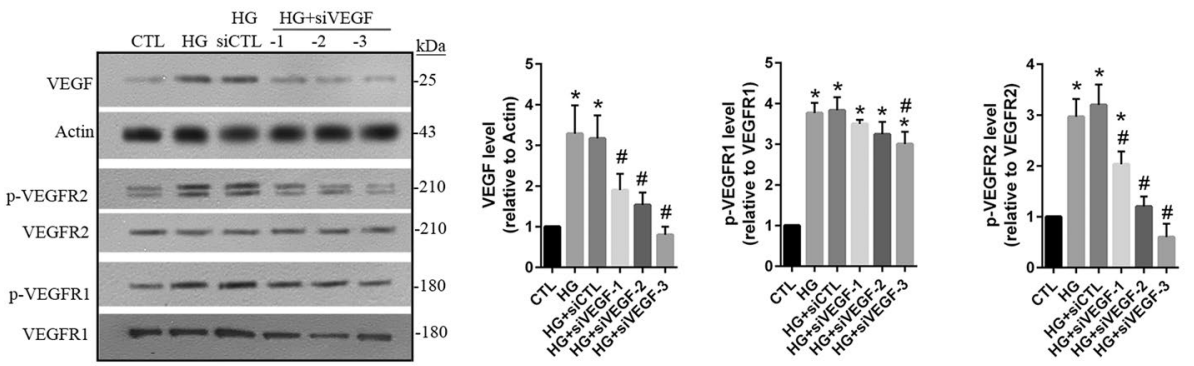

b
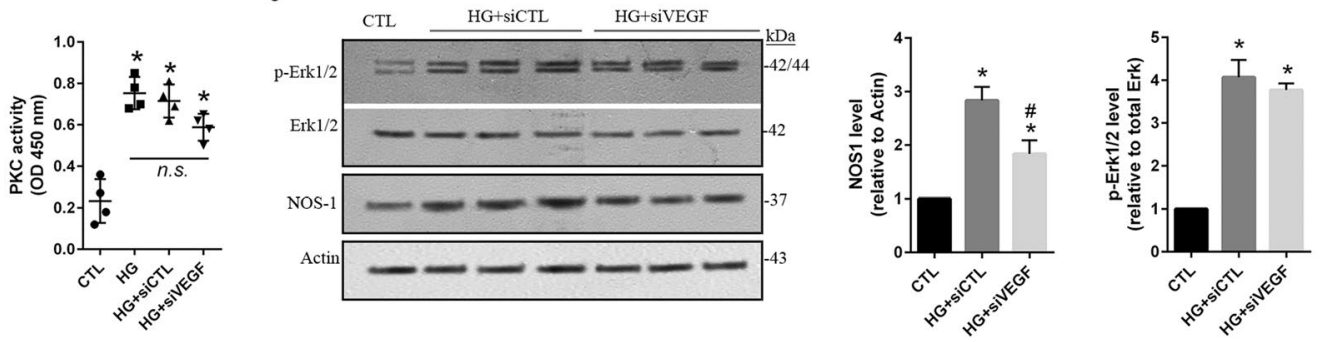

d
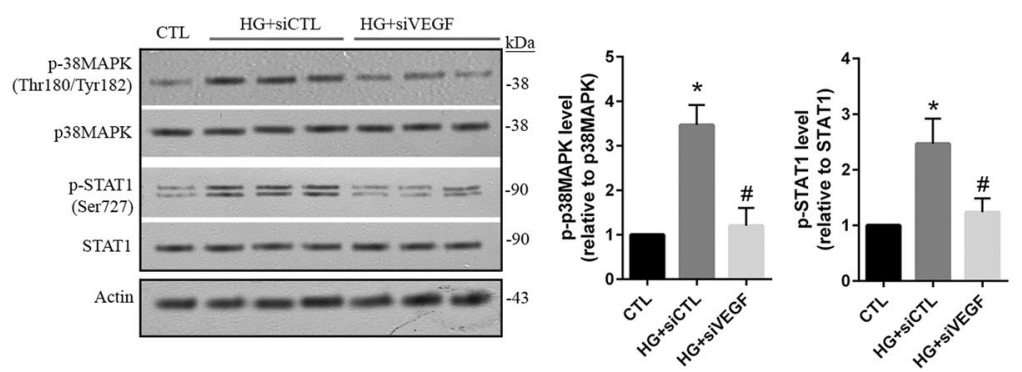

e

f

Figure 5. VEGF-mediated activation of the p38MAPK/STAT1 signaling via VEGFR2 involves increase of apoptosis in HG-treated hRECs. (a). In cultured hRECs, the siRNA that targets to human VEGFA gene was introduced at 3 different concentrations (siVEGF-1: $5 \mathrm{nM}$, siVEGF-2: $10 \mathrm{nM}$, siVEGF-3: $20 \mathrm{nM}$ ). Control siRNA (siCTL, $20 \mathrm{nM}$ ) that does not target any human gene was used as the transfection control. $24 \mathrm{~h}$ after transfection, cells were exposed to high glucose (HG, $25 \mathrm{uM})$ for $24 \mathrm{~h}$. Cells were then lysed and total cellular protein was extracted for immunoblotting with anti-VEGF, anti-phospho-VEGFR1 ${ }^{\text {Tyr1213 }}$, and anti-phosphoVEGFR2Tyr1175 antibodies. (b). In VEGF knockdown cell (siVEGF, $20 \mathrm{nM}$ ), effect of HG on PKC activity was evaluated using PKC Kinase Activity Assay. (c). In VEGF knockdown cell (siVEGF, $20 \mathrm{nM}$ ), immunoblot assay was performed to investigate the effects of HG on the level of phospho-Erk1/2 $2^{\text {Tyr202/Tyr185 }}$ and NOS1 expression. (d). In VEGF knockdown cell (siVEGF, $20 \mathrm{nM}$ ), immunoblot assay was performed to show the effects of $\mathrm{HG}$ on the level of phospho-p38MAPK ${ }^{\text {Tyr180/Tyr182 }}$ and phospho-STAT1 ${ }^{\text {Ser727. }}$. (e). In VEGF knockdown cell (siVEGF, $20 \mathrm{nM}$ ), apoptotic cell death was determined following HG treatment. (f). Cells were grown on Transwell filters, and VEGF was knocked down using siVEGF $(20 \mathrm{nM})$. Following HG treatment, the permeability of monolayer cells was measured. Data are presented mean $\pm S D$. $a, c$ and $d: n=3 ; B: n=4 ; e$ and $f: n=5$. * $p<0.05$ vs CTL, ${ }^{\#} p<0.05$ vs HG or HG + siCTL; n.s. non-significance. In $c$ and d, stripped membranes from phospho-Erk, phosphop38MAPK, and phospho-STAT1 were reblotted for total Erk, p38MAPK, and STAT1, respectively.

Notably, only in vitro experiments were performed in this study since the current condition does not allow to do in vivo explorations. The data from human endothelial cultures are unlikely to address a complex in vivo issue that involves multiple cell-types. Therefore, it is well worth of investigating and validating the present findings in an in vivo DR model. Recently, increased PIGF and VEGF was detected in a new diabetic mouse strain, Akita, and PlGF deletion in these mice prevented diabetic-induced retinal cell death, capillary degeneration, pericyte loss, and blood-retinal barrier breakdown ${ }^{41}$. This suggests that PlGF is critical for the development of DR, and its genetic deletion protects the retina from diabetic damage. The conclusion is in consistent with our findings that HG increased both PlGF and VEGF expressions, and PlGF knockdown mainly prevented HG-induced hyperpermeability in hRECs. Nevertheless, we did not find that PlGF knockdown had significant effect on HG-induced cell death. Additionally, PlGF knockout inhibited the HIF1 $\alpha$-VEGF signaling pathway in the Akita mice retinas, presenting with reduction of phospho-VEGFR1, phospho-VEGFR2 and phospho-eNOS compared to wild type (WT) Akita mice ${ }^{41}$. In our in vitro model, PlGF knockdown predominantly decreased the levels of phospho-VEGFR1 and total NOS1, but not phospho-VEGFR2. The Akt pathway is a signal transduction pathway that promotes survival and growth in response to extracellular signals. Akt activity was reduced in the WT Akita 
$\mathrm{a}$
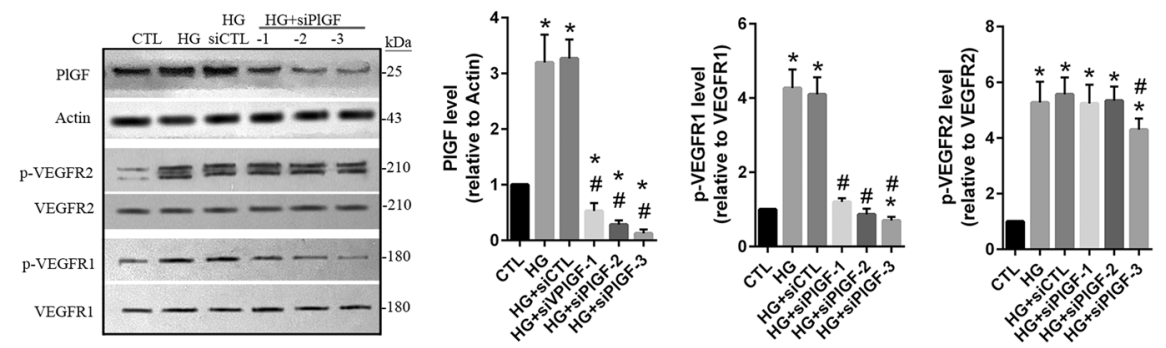

b

c

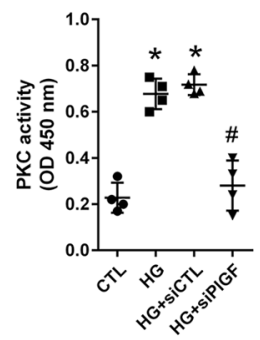

d

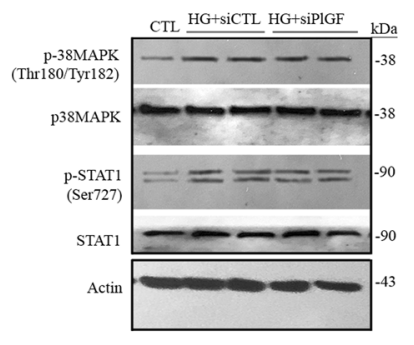

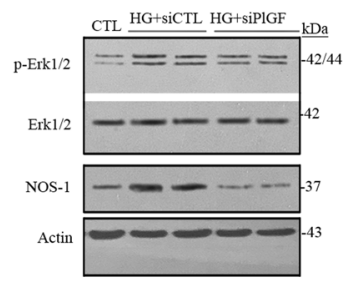

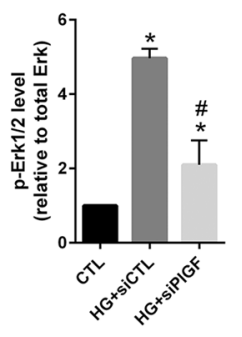

$\mathrm{e}$

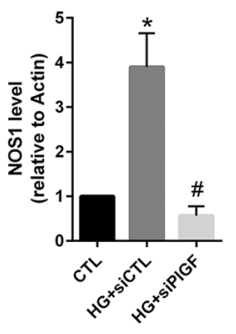

$\mathrm{f}$

Figure 6. PlGF-mediated activation of the PKC-Erk1/2-NOS axis via VEGFR1 is mainly related to increase of permeability in HG-treated hRECs. (a). In cultured hRECs, the siRNA that targets to human PlGF gene was introduced at different concentration (siPlGF-1: $5 \mathrm{nM}$, siPlGF-2: $10 \mathrm{nM}$, siPlGF-3: $20 \mathrm{nM}$ ). Control siRNA ( $\mathrm{siCTL}, 20 \mathrm{nM}$ ) that does not target any human gene was used as the transfection control. $24 \mathrm{~h}$ after transfection, cells were exposed to high glucose ( $\mathrm{HG}, 25 \mathrm{uM})$ for $24 \mathrm{~h}$. Cells were then lysed and total cellular protein was extracted for immunoblotting with anti-PlGF, anti-phospho-VEGFR1 ${ }^{\text {Tyr1213 }}$, and anti-phospho-VEGFR2 ${ }^{\text {Tyr1175 }}$ antibodies. (b). In PlGF knockdown cell (siPlGF, $20 \mathrm{nM}$ ), effect of HG on PKC activity was evaluated using PKC Kinase Activity Assay. (c). In PlGF knockdown cell (siPlGF, 20 nM), immunoblot assay was performed

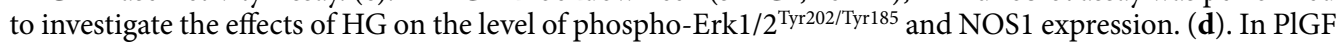
knockdown cell (siPlGF, $20 \mathrm{nM}$ ), immunoblot assay was performed to show the effects of HG on the level

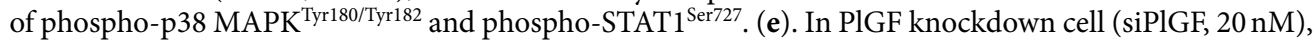
apoptotic cell death was determined following HG treatment. (f). Cells were grown on Transwell filters, and PlGF was knocked down using siPlGF $(20 \mathrm{nM})$. Following HG treatment, the permeability of monolayer cells was measured. Data are presented mean $\pm S D$. $a, c$ and $d: n=3 ; b: n=4 ;$ e and $f: n=5$. ${ }^{*} p<0.05$ vs CTL, ${ }^{\#} p<0.05$ vs HG or HG + siCTL; n.s. non-significance. In $c$ and d, stripped membranes from phospho-Erk, phosphop38MAPK, and phospho-STAT1 were reblotted for total Erk, p38MAPK, and STAT1, respectively.

mice, while increased dramatically in PlGF knockout Akita mice ${ }^{41}$. However, our and other studies ${ }^{26,33,34}$ showed a significant activation of Akt in HG-treated hRECs (data not shown). These discrepancies may be resulted from the differences between the in vivo and the in vitro experiments. The other thing is that global knockout of PlGF was obtained in the Akita mice, which may be the other potential reason for the discrepancies. Therefore, future work is needed to perform specific knockout of PlGF in the retinal endothelial cells in the Akita mice in order to determine the distinct role and downstream signaling of PIGF. Moreover, interaction of pericytes with endothelial cells is important for endothelial cell viability. The role and proximal signaling of VEGF and PlGF need be further investigated in HG-induced hRECs injuries in the presence of pericytes.In this study, activation levels of p38MAPK/STAT1 were significantly suppressed by knockdown of VEGF but not PlGF in HG-treated hRECs. Our data show that VEGF knockdown selectively inhibited activation of VEGFR2 in cultured hRECs under HG condition. HG-induced overactivation of p38MAPK/STAT1 signaling was significantly prohibited in VEGF knockdown cell. Nevertheless, VEGF knockdown showed no remarkable influences on PKC activity as well as phosphorylated Erk1/2 and NOS1 expressions in HG treated hRECs. In consistent, apoptotic cell death, but not hyperpermeability, was significantly decreased by VEGF knockdown in HG-treated hRECs. It was reported that increased VEGF and p38MAPK activation were also related to induction of apoptosis in hRECs under HG 


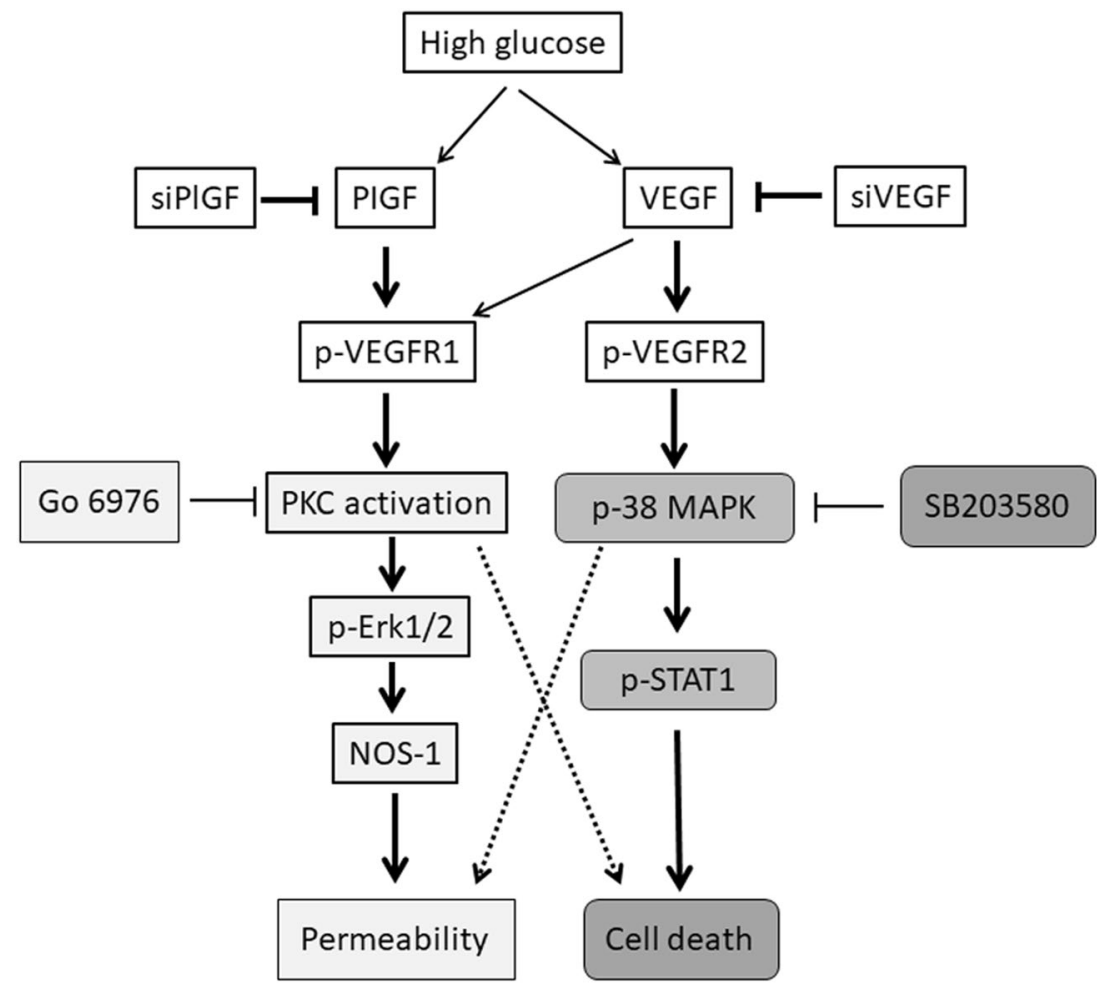

Figure 7. The schematic of the proposed VEGF and PlGF signaling in HG-induced hRECs injuries. Hyperpermeability and increased cell death was detected in HG-treated hRECs. Following HG treatment, expression of VEGF and PlGF was upregulated, phosphorylation of VEGFR1 and VEGFR2 as well as PKC activity and NOS1 abundance were increased, and activation of Erk1/2, p38MAPK and STAT1 were increased. Inhibition of PKC activity predominantly prevented activation of Erk1/2 and decreased NOS 1 level and permeability, while did not affect apoptotic cell death. Inhibition of p38MAPK dramatically inhibited activation of STAT1 and decreased cell death, while did not show significant effect on permeability. Knockdown of VEGF mainly decreased phosphorylation of VEGFR2 and p38MAPK as well as cell death; knockdown of PlGF mainly decreased phosphorylation of VEGFR1 as well as PKC activity and permeability. Go 6976: PKC inhibitor, SB203580: $338 M A P K$ inhibitor.

conditions ${ }^{33}$. Therefore, our findings suggest that VEGF predominantly increased the activation of p38MAPK/ STAT1 via VEGFR2, thus leading to induction of apoptotic cell death in HG-treated hRECs (Fig. 7).

Taken together, we separated the downstream signaling of VEGF and PlGF in HG-induced injuries of hRECs. In HG-treated hRECs, VEGF-mediated activation of p38MAPK/STAT1 signaling via binging to VEGFR2 mainly led to apoptosis, while PlGF-mediated activation of PKC-Erk1/2-NOS1 signaling via binding to VEGFR1 mainly resulted in hyperpermeability.

\section{Materials and Methods}

Primary antibodies. The following primary antibodies were used in this study: mouse anti-bovine serum albu$\min (B 2901,1: 1,000)$, rabbit anti-NOS1 (SAB4502010, 1: 500), and mouse anti- $\beta$-actin (A2228, 1: 10,000) antibodies from Sigma-Aldrich (St. Louis, MO, USA); rabbit anti-caspase3 (ab32351, 1: 1,000), rabbit anti-active caspase 3 (ab2302, 1: 500), rabbit anti-Bcl2 (ab59348, 1: 1,000), rabbit anti-PKC (ab19031, 1: 500), mouse anti-Erk1/2 (ab54230, 1: 1,000), rabbit anti-phospho-Erk1 ${ }^{\text {Tyr202 }}$ and $\operatorname{Erk}^{\text {Tyr185 }}(\mathrm{ab} 201015,1: 1,000)$, rabbit-anti-Flit1/VEGFR1 (ab2350, 1: 500), rabbit anti-KDR/VEGFR2 (ab39256, 1: 600), rabbit anti-human CD31 (ab28364, 1: 250), rabbit anti-Von Willebrand Factor antibody (ab6994, 1: 250), and rabbit anti-PlGF (ab9542, 1: 500) antibodies from Abcam (Cambridge, MA, USA); mouse anti-VEGF (cat. no. 05-1117, 1: 1,000) and rabbit anti-phospho-VEGFR1 ${ }^{\text {Tyr1213 }}$ (cat. no. 07-758, 1: 500) antibodies from EMD Millipore (Burlington, MA, USA); rabbit anti-phospho-VEGFR2 ${ }^{\text {Tyr1175 }}$ (\#2478, 1: 800), rabbit

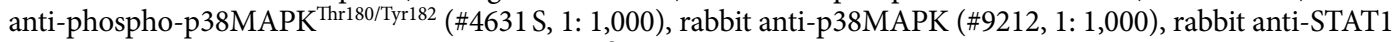
(\#9172, 1: 500), and rabbit anti-phospho-STAT1 ${ }^{\text {Ser727 }}(\# 8826,1: 500)$ antibodies from Cell Signaling Technology (Danvers, MA, USA). Alexa Fluo 594 goat anti-rabbit IgG antibody (Cat. no. A-11037, 1: 2,000, Invitrogen; Thermo Fisher Scientific, Inc., Waltham, MA USA) was used for indirect immunofluorescence staining assay.

Cell culture and treatment. Human microvascular retinal endothelial cell line (ACBRI 181, hRECs) was obtained from Cell Systems (Kirkland, WA, USA). As previously described ${ }^{33}$, hRECs were grown in fibronectin-coated plate in Dulbecco's Modified Eagle's Medium (cat. no. 11885-084) supplemented with 10\% fetal calf serum (cat. no. 16000-044) and $100 \mathrm{U} / \mathrm{ml}$ of penicillin/streptomycin (cat. no. 15140-122; Gibco; Thermo Fisher Scientific, Inc.). To maintain uniform conditions, all experiments were performed by using passage 4-6 
hRECs in this study. Cultured hRECs were also identified by stain of CD13 and von Willebrand Factor (vWF) ${ }^{34}$; the positive staining of CD13 and vWF has been provided (Supplemental Fig. 1).

hRECs were cultured in the presence of $25 \mathrm{mM}$ of glucose (cat. no. G8270, Sigma-Aldrich) or mannitol (cat. no. PHR1007, Sigma-Aldrich) for different time periods. In some cases, $10 \mu \mathrm{g} / \mathrm{ml}$ bovine serum albumin (BSA; B8667, Sigma-Aldrich), 500 nM PKC inhibitor Go 6976 (cat. no. 2253/1, R\&D Systems; Minneapolis, MN), and $10 \mu \mathrm{M}$ p38 MAPK inhibitor SB203580 (\#5633, Cell Signaling Technology) was added to the medium alone or in appropriate combination as indicated.

Cell death detection. Apoptotic cell death was determined using the Cell Death Detection ELISA kit (cat. no. 11774425 001; Roche; Mannheim, Germany) as described previously ${ }^{21}$. Triplicate was used in each condition. The absorbance at $405 \mathrm{~nm}$ and $490 \mathrm{~nm}$ (reference wavelength) was determined with a microplate reader (Bio-Tec Instruments, Winooski, VT, USA). Signals in the wells containing the substrate only were used as the background and subtracted.

ELISA assay. The amounts of VEGF and PlGF in cell culture supernatants were measured using Human VEGF ELISA Kit (ab100662, Abcam) and Human PlGF Quantikine ELISA Kit (cat. no. DPG00, R\&D Systems) according to the manufacturer's procedure, respectively.

Permeability detection assay. hRECs were seeded on polyethylene membrane insert (a pore size of 1.0 $\mu \mathrm{m})$ in the Transwell chamber and treated with HG or mannitol for the indicated time periods. FITC-conjugated bovine serum albumin (BSA; cat. no. A9771, Sigma-Aldrich) was added at the final concentration of $10 \mu \mathrm{g} / \mathrm{ml}$ in each upper chamber, whereas the lower chamber was filled with $500 \mu \mathrm{l}$ medium alone. Transwell plates were incubated for $30 \mathrm{~min}$ at $37^{\circ} \mathrm{C}$. The solution in lower chamber containing permeabilized FITC-BSA was collected to measure the fluorescence intensity using microplate reader (Bio-Tec Instruments) with the excitation and emission wavelengths of 490 and $525 \mathrm{~nm}$, respectively. The amount of permeabilized FITC-BSA $(\mu \mathrm{g} / \mathrm{ml})$ was then calculated according to the standard concentration curve.

RT-PCR. Quantitative real time PCR was performed as described previously ${ }^{21}$. Briefly, total RNA was extracted and quantitated. Totally, $2 \mu \mathrm{g}$ of RNA was transcribed to the cDNA with SuperScript ${ }^{\mathrm{TM}}$ III First-Strand Synthesis Kit (cat. no. 11752-050; Thermo Fisher Scientific, Inc.). Quantitative PCR was then performed using a 7500 fast Real-Time PCR system (Applied Biosystems; Thermo Fisher Scientific, Inc.). The SYBR Green PCR Master Mix (cat. no. 1725270; Bio-Rad, Hercules, CA, USA) was used, including $1.5 \mu \mathrm{l}$ of cDNA and $0.2 \mu \mathrm{M}$ of specific primer pairs (VEGF: forward $5^{\prime}$-atcttcaagccatcctgtgtgc- $3^{\prime}$, reverse $5^{\prime}$-caaggcccacagggattttc- $3^{\prime}$; PlGF: forward $5^{\prime}$-gttcagcccatcctgtgtct- $3^{\prime}$, reverse $5^{\prime}$-ttaggagctgcatggtgaca-3'; GAPDH: forward $5^{\prime}$-tgtgtccgtcgtggatctga- $3^{\prime}$, reverse $5^{\prime}$-cctgcttcaccaccttcttga- $3^{\prime}$ ). Two-step PCR program was run: initial denaturation at $95^{\circ} \mathrm{C}$ for $10 \mathrm{~min} ; 40$ cycles of $95^{\circ} \mathrm{C}$ for $15 \mathrm{sec}$ and $55^{\circ} \mathrm{C}$ for $45 \mathrm{sec}$. The relative expression levels of VEGF and PlGF targeted genes were calculated using the $2^{-\Delta \Delta \mathrm{Cq}}$ method and normalized to the house-keeping gene GAPDH. The fold change over the controls was presented in this study.

Knockdown assay. To delete the expressions of VEGF and PlGF at mRNA levels, siRNA-VEGFA (sc-29520; Santa Cruz, Dallas, TX, USA) and siRNA-PlGF (sc-44027; Santa Cruz) were introduced to hRECs at the final concentration of 5, 10 or $20 \mathrm{nM}$ with RNAiMAX (cat. no. 13778-075; Invitrogen; Thermo Fisher Scientific, Inc.). Non-targeted siRNA (sc-37007, Santa Cruz) was used as the controls. Cells were then collected after 48 hours. Knockdown efficiency of the siRNAs was evaluated in hRECs under normoglycemia condition (Supplemental Fig. 2).

Western blot assay. As described previously ${ }^{21}$, western blot assay was performed. Briefly, cells were lysed in RIPA buffer (20 mM Tris-HCl pH 7.5, 150 mM NaCl, 1 mM EDTA, 1 m M EGTA, 1\% NP-40, $1 \%$ sodium deoxycholate, $2.5 \mathrm{mM}$ sodium pyrophosphate, $1 \mathrm{mM} \beta$-glycerophosphate, $1 \mathrm{mM} \mathrm{Na}_{3} \mathrm{VO}_{4}$, and $1 \mu \mathrm{g} / \mathrm{ml}$ leupeptin, $1 \mathrm{mM}$ PMSF), and total cellular protein was extracted and quantitated. Equal amount of protein $(75 \mu \mathrm{g})$ was separated using 7.5 or $12.5 \%$ sodium dodecyl sulfate polyacrylamide gel electrophoresis, and transferred to nitrocellulose membrane (Abcam). The membrane was blocked for $1 \mathrm{~h}$ in $5 \%$ low-fat milk or BSA prepared in Tris-buffered saline containing $0.1 \%$ Tween-20 (TBST), and then incubated for over-night with the indicated primary antibodies followed by incubation with HRP-conjugated goat anti-rabbit or goat anti-mouse IgG (1: 10,000; cat. no. G-21234, G-21040; Invitrogen; Thermo Fisher Scientific, Inc.) for $1 \mathrm{~h}$ at room temperature. After 5 washes with TBST, the blots were developed using ECL Western Blotting Substrate (cat. no. 32109; Pierce; Thermo Fisher Scientific, Inc.). To blot another $\mathrm{Ab}$ in the same membrane, the membrane was stripped by incubating the membrane in the stripping buffer (cat. no. 46430, Thermo Fisher Scientific, Inc.) for $15 \mathrm{~min}$ at room temperature; then washed three times with TBST, blocked for $1 \mathrm{~h}$ in $5 \% \mathrm{BSA}$, and another $\mathrm{Ab}$ was then applied for overnight at $4^{\circ} \mathrm{C}$. The specific band was detected and the intensities were quantitated by using Image J (Version $1.51 \mathrm{~s}$; National Institute of Health, Rockville, MD, USA).

PKC activity assay. PKC activity was evaluated using PKC Kinase Activity Assay Kit according to the manufacturer's instructions (ab139437, Abcam). Briefly, $50 \mu \mathrm{g}$ total proteins in $30 \mu \mathrm{l}$ were added to PKC Substrate Microtiter Plate. Dilution buffer was used as Blank control. $10 \mu \mathrm{l}$ of ATP $(1 \mathrm{mg} / \mathrm{ml})$ was added to each well, and incubated at $30^{\circ} \mathrm{C}$ for $2 \mathrm{~h} .40 \mu \mathrm{l}$ of the Phospho-specific Substrate Antibody was added and incubated at room temperature for $1 \mathrm{~h}$. After four washes, $40 \mu \mathrm{l}$ of HRP-conjugate anti-rabbit IgG (1: 1,000) was added to each well, and incubated at room temperature for $30 \mathrm{~min}$. After 4 washes, $60 \mu \mathrm{l}$ of the TMB Substrate was added, and 
incubated for $45 \mathrm{~min}$. Finally, $20 \mu \mathrm{l}$ of the Stop Solution was added. The absorbance was measured at $450 \mathrm{~nm}$ with Microplate reader (Bio-Tec Instruments). The PKC kinase activity was expressed by the value of OD450.

Statistical analysis. Data are shown as mean \pm S.D. One-way ANOVA and Two-Way ANOVA were used to perform statistical analysis (GraphPad Prism 6.0; La Jolla, CA, USA). The value of $p$ less than and/or equal to 0.05 was considered to have significant differences.

Received: 18 March 2019; Accepted: 29 September 2019;

Published online: 25 October 2019

\section{References}

1. Yau, J. W. et al. Global prevalence and major risk factors of diabetic retinopathy. Diabetes Care. 35(3), 556-564 (2012).

2. Leasher, J. L. et al. Global Estimates on the Number of People Blind or Visually Impaired by Diabetic Retinopathy: A Meta-analysis From 1990 to 2010. Diabetes Care. 39(9), 1643-1649 (2016).

3. Zhang, G., Chen, H., Chen, W. \& Zhang, M. Prevalence and risk factors for diabetic retinopathy in China: a multi-hospital-based cross-sectional study. Br J Ophthalmol. 101(12), 1591-1595 (2017).

4. Capitão, M. \& Soares, R. Angiogenesis and Inflammation Crosstalk in Diabetic Retinopathy. J Cell Biochem. 117(11), 2443-2453 (2016).

5. Crawford, T. N., Alfaro, D. V. 3rd, Kerrison, J. B. \& Jablon, E. P. Diabetic retinopathy and angiogenesis. Curr Diabetes Rev. 5(1), 8-13 (2009).

6. Zhou, L. et al. Lonicerae Japonicae Flos attenuates diabetic retinopathy by inhibiting retinal angiogenesis. J Ethnopharmacol. 189, 117-125 (2016)

7. Tremolada, G. et al. The role of angiogenesis in the development of proliferative diabetic retinopathy: impact of intravitreal antiVEGF treatment. Exp Diabetes Res. 2012, 728325 (2012).

8. Melincovici, C. S. et al. Vascular endothelial growth factor (VEGF) - key factor in normal and pathological angiogenesis. Rom J Morphol Embryol. 59(2), 455-467 (2018).

9. Selim, K. M., Sahan, D., Muhittin, T., Osman, C. \& Mustafa, O. Increased levels of vascular endothelial growth factor in the aqueous humor of patients with diabetic retinopathy. Indian J Ophthalmol. 58(5), 375-379 (2010).

10. Mesquita, J. et al. Vascular endothelial growth factors and placenta growth factor in retinal vasculopathies: Current research and future perspectives. Cytokine Growth Factor Rev. 39, 102-115 (2018).

11. Zhang, F. et al. VEGF-B is dispensable for blood vessel growth but critical for their survival, and VEGF-B targeting inhibits pathological angiogenesis. Proc Natl Acad Sci USA 106(15), 6152-6157 (2009).

12. Mitamura, Y. et al. Vitreous levels of placenta growth factor and vascular endothelial growth factor in patients with proliferative diabetic retinopathy. Diabetes Care. 25(12), 2352 (2002).

13. Ando, R. et al. Aqueous humour levels of placental growth factor in diabetic retinopathy. Acta Ophthalmol. 92(3), e245-e246 (2014).

14. Al Kahtani, E. et al. Vitreous levels of placental growth factor correlate with activity of proliferative diabetic retinopathy and are not influenced by bevacizumab treatment. Eye (Lond). 31(4), 529-536 (2017).

15. Pipp, F. et al. VEGFR-1-selective VEGF homologue PlGF is arteriogenic: evidence for a monocyte-mediated mechanism. Circ Res 92(4), 378-385 (2003).

16. Babiak, A. et al. Coordinated activation of VEGFR-1 and VEGFR-2 is a potent arteriogenic stimulus leading to enhancement of regional perfusion. Cardiovasc Res. 61(4), 789-795 (2004).

17. Tchaikovski, V., Fellbrich, G. \& Waltenberger, J. The molecular basis of VEGFR-1 signal transduction pathways in primary human monocytes. Arterioscler Thromb Vasc Biol. 28(2), 322-328 (2008).

18. Berridge, M. J. Inositol trisphosphate and calcium signalling. Nature. 361, 315-325 (1993).

19. Sironi, J. J. \& Ouchi, T. STAT1-induced apoptosis is mediated by caspases 2, 3, and 7. J Biol Chem. 279(6), 4066-4074 (2004).

20. Abdel-Magid, A. F. Plasma Kallikrein Inhibitors for the Treatment of Retinal Vascular Permeability Associated with Diabetic Retinopathy and Diabetic Macular Edema. ACS Med Chem Lett. 8(8), 776-777 (2017).

21. Jiao, W. et al. Activation of the Notch-Nox4-reactive oxygen species signaling pathway induces cell death in high glucose-treated human retinal endothelial cells. Mol Med Rep. 19(1), 667-677 (2019).

22. Liu, J. et al. Palmitate promotes autophagy and apoptosis through ROS-dependent JNK and p38 MAPK. Biochem Biophys Res Commun. 463(3), 262-267 (2015).

23. Kovarik, P. et al. Stress-induced phosphorylation of STAT1 at Ser727 requires p38 mitogen-activated protein kinase whereas IFNgamma uses a different signaling pathway. Proc Natl Acad Sci USA 96(24), 13956-13961 (1999).

24. Antonetti, D. A., Klein, R. \& Gardner, T. W. Diabetic retinopathy. N Engl J Med. 366(13), 1227-1239 (2012).

25. Cunha-Vaz, J., Ribeiro, L. \& Lobo, C. Phenotypes and biomarkers of diabetic retinopathy. Prog Retin Eye Res. 41, 90-111 (2014)

26. Xu, X. H., Zhao, C., Peng, Q., Xie, P. \& Liu, Q. H. Kaempferol inhibited VEGF and PGF expression and in vitro angiogenesis of hRECs under diabetic-like environment. Braz J Med Biol Res. 50(3), e5396 (2017).

27. Wu, H. M., Yuan, Y., Zawieja, D. C., Tinsley, J. \& Granger, H. J. Role of phospholipase C, protein kinase C, and calcium in VEGF induced venular hyperpermeability. Am J Physiol Heart Circ Physiol. 276, H535-H542 (1999).

28. Lal, B. K., Varma, S., Pappas, P. J., Hobson, R. W. \& Dura'n, W. N. VEGF increases permeability of the endothelial cell monolayer by activation of PKB/akt, endothelial nitric-oxide synthase, and MAP kinase pathways. Microvasc Res. 62, 252-262 (2001).

29. Cobb, M. H. MAP kinase pathways. Prog Biophys Mol Biol. 71, 479-500 (1999).

30. Takahashi, T., Ueno, H. \& Shibuya, M. VEGF activates protein kinase C-dependent, but Ras-independent Raf-MEK-MAP kinase pathway for DNA synthesis in primary endothelial cells. Oncogene. 18, 2221-2230 (1999).

31. Berridge, M. J. Inositol trisphosphate and calcium signalling. Nature. 361, 315-325 (1993)

32. Breslin, J. W., Pappas, P. J., Cerveira, J. J., Hobson, R. W. II \& Durán, W. N. VEGF increases endothelial permeability by separate signaling pathways involving ERK-1/2 and nitric oxide. Am J Physiol Heart Circ Physiol. 284(1), H92-H100 (2003).

33. Zhang, L., Zhang, Z. K. \& Liang, S. Epigallocatechin-3-gallate protects retinal vascular endothelial cells from high glucose stress in vitro via the MAPK/ERK-VEGF pathway. Genet Mol Res. 15(2), https://doi.org/10.4238/gmr.15027874 (2016).

34. Chen, X. et al. KH902 suppresses high glucose-induced migration and sprouting of human retinal endothelial cells by blocking VEGF and PIGF. Diabetes Obes Metab. 15(3), 224-233 (2013).

35. Blank, V. C., Peña, C. \& Roguin, L. P. STAT1, STAT3 and p38MAPK are involved in the apoptotic effect induced by a chimeric cyclic interferon-alpha2b peptide. Exp Cell Res. 316(4), 603-614 (2010).

36. Zhao, B., Cai, J. \& Boulton, M. Expression of placenta growth factor is regulated by both VEGF and hyperglycaemia via VEGFR-2. Microvasc Res. 68, 239-246 (2004).

37. Fujii, T. et al. VEGF function for upregulation of endogenous PIGF expression during FGF-2-mediated therapeutic angiogenesis. Atherosclerosis. 200(1), 51-57 (2008). 
38. Zhang, X., Bao, S., Lai, D., Rapkins, R. W. \& Gillies, M. C. Intravitreal triamcinolone acetonide inhibits breakdown of the bloodretinal barrier through differential regulation of VEGF-A and its receptors in early diabetic rat retinas. Diabetes. 57, 1026-1033 (2008).

39. Mitsui, T. et al. Upregulation of Angiogenic Factors via Protein Kinase $C$ and Hypoxia-induced Factor-1 $\alpha$ Pathways under Highglucose Conditions in the Placenta. Acta Med Okayama. 72(4), 359-367 (2018).

40. Deissler, H. L., Deissler, H., Lang, G. K. \& Lang, G. E. VEGF but not PIGF disturbs the barrier of retinal endothelial cells. Exp Eye Res. 115, 162-171 (2013).

41. Huang, H. et al. Deletion of placental growth factor prevents diabetic retinopathy and is associated with Akt activation and HIF1 $\alpha-$ VEGF pathway inhibition. Diabetes. 64(1), 200-212 (2015).

\section{Acknowledgements}

This work was supported by the Key Research and Development Program of Shandong Province of China (2017GSF18178 to WX), the National Natural Science Foundation of China (61572300, 81871508 and 61773246 to YZ), the Taishan Scholar Program of Shandong Province of China (TSHW201502038 to YZ), the Major Program of Shandong Province Natural Science Foundation (ZR2018ZB0419 to YZ), and the Shandong Nature Science Foundation (ZR2019MH111 and ZR2017MH021 to BZ and AM).

\section{Author contributions}

Study conception and design: W.J., J.J., Q.F., A.M. and B.Z., Experiment and data analysis: W.J., J.J., W.X. and W.B., Statistical analyses: W.J., W.B. and Y.Z., Manuscript preparation: J.W., Critical revision: J.W. and B.Z.

\section{Competing interests}

The authors declare no competing interests.

\section{Additional information}

Supplementary information is available for this paper at https://doi.org/10.1038/s41598-019-51603-0.

Correspondence and requests for materials should be addressed to A.M. or B.Z.

Reprints and permissions information is available at www.nature.com/reprints.

Publisher's note Springer Nature remains neutral with regard to jurisdictional claims in published maps and institutional affiliations.

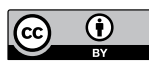

Open Access This article is licensed under a Creative Commons Attribution 4.0 International License, which permits use, sharing, adaptation, distribution and reproduction in any medium or format, as long as you give appropriate credit to the original author(s) and the source, provide a link to the Creative Commons license, and indicate if changes were made. The images or other third party material in this article are included in the article's Creative Commons license, unless indicated otherwise in a credit line to the material. If material is not included in the article's Creative Commons license and your intended use is not permitted by statutory regulation or exceeds the permitted use, you will need to obtain permission directly from the copyright holder. To view a copy of this license, visit http://creativecommons.org/licenses/by/4.0/.

(C) The Author(s) 2019 